\title{
Putting the brakes on arthritis: can suppressors of cytokine signaling (SOCS) suppress rheumatoid arthritis?
}

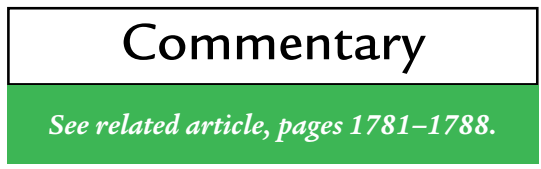

\author{
Robert Rottapel ${ }^{1,2}$ \\ ${ }^{1}$ St. Michael's Hospital, Toronto, Ontario, Canada \\ ${ }^{2}$ Departments of Immunology, Medical Biophysics, and Medicine, University of Toronto, Toronto, Canada \\ Address correspondence to: Robert Rottapel, Departments of Immunology, Medical Biophysics, and Medicine, \\ University of Toronto, Ontario Cancer Institute, 610 University Avenue, Toronto, Ontario, Canada M5G 2M9. \\ Phone: (416) 946-2233; Fax: (416) 946-2984; E-mail: rottapel@uhnres.utoronto.ca.
}

J. Clin. Invest. 108:1745-1747 (2001). DOI:10.1172/JCI200114661.

\begin{abstract}
During the immune response, as in a great symphony, timing is everything. Imagine the disastrous night of November 3, 1822, when Beethoven conducted a revival of his popular opera Fidelio. The musicians played their parts from the score perfectly but missed every cue of the great master, who was deaf to everything but his own imagination. The evening ended in chaos. The critical role for molecular cues firing at the right time is also true for the cellular players of the immune network, a system stitched together by the interplay of multiple cytokines.
\end{abstract} These peptide signals coordinate cell survival, migration, proliferation, and activation of cellular effector function. Not only do the right cytokine signals have to be initiated at the right time, but the duration of each signal must be perfectly controlled. Otherwise, disasters like autoimmunity or malignancies of the hematopoietic system can result.

The longevity of cytokine signals transduced by the JAK/STAT pathway is regulated, in part, by a family of endogenous JAK kinase inhibitor proteins referred to as suppressors of cytokine signaling (SOCS) $(1,2)$. The SOCS family contains eight members of related proteins that share a common modular organization of an $\mathrm{SH} 2$ domain followed by a SOCS-box (reviewed in ref. 3). Two members of this family, SOCS-1 and SOCS-3, are potent inhibitors of JAK kinase family members. Both SOCS-1 and SOCS-3 bind to the positive regulatory tyrosine in the activation loop of JAK kinases through their respective $\mathrm{SH} 2$ domains, thereby occluding the accessibility of the active site to substrates (4). The tis- sue expression patterns of SOCS proteins are complex and distinct for each family member.

Several SOCS members are earlyresponse genes for a variety of cytokines and growth factors. For example, IL-6 induces SOCS-1, erythropoietin induces SOCS-2, and TNF- $\alpha$ induces SOCS-3, while IFN- $\gamma$ potently induces all three family members. The transcriptional regulation of these SOCS family members appears to be under the exquisite control of the STAT transcription factors. Since SOCS- 1 and SOCS-3 expression is tightly coupled to upstream cytokine signaling events it appears that they function as components of a negative feedback loop to dynamically terminate cytokine-mediated signals. Support for this model has been borne out by analysis of mice lacking SOCS proteins. SOCS-1-deficient mice die 3 weeks after birth as a result of unbridled IFN- $\gamma$ signaling leading to widespread myelomonocytic infiltration of visceral organs and skin $(5,6)$. Socs $1^{-1-} / I F N-\gamma^{\dagger /-}$ mice develop an autoimmune polymyositis about 160 days after birth (7). Deficiency in SOCS-3 in mice results in embryonic lethality at day 12-16 and fetal liver erythrocytosis (8). For more on the central role of SOCS-3 in neuroendocrine cross-regulation, see the Perspective by Auernhammer and Melmed in this issue (9).

\section{Cytokine dysregulation \\ in rheumatoid arthritis}

Could disordered temporal signaling of cytokines contribute to pathogenesis of human diseases involving the immune system? One possible example may be rheumatoid arthritis (RA). RA, a chronic disease of joint inflammation, is endemic in our society, affecting approximately $1 \%$ of the population. Although the etiology underlying RA remains unknown, it is clear that inflammatory cytokine circuits are established in the synovial cells lining the joint in genetically susceptible individuals. One of the master cytokines in this cascade appears to be TNF- $\alpha$. Dysregulated expression of TNF- $\alpha$ in experimental animals is sufficient to cause destructive arthritis (10), and blocking TNF- $\alpha$ with high-affinity antibodies or soluble receptors dramatically improves the clinical status of patients $(11,12)$. Other cytokines may also be involved in the pathogenesis of RA, including IL-1 and IL-6. Mice lacking IL-6 are not susceptible to antigen-induced arthritis, suggesting that IL- 6 might be another important player in the rheumatoid cytokine network $(13,14)$.

In this issue of the JCI, a study from the laboratory of Akihiko Yoshimura provides evidence for abnormal cytokine signaling in an animal model of inflammatory synovitis and reports on the utility of forced expression of SOCS- 3 by adenovirus gene transfer in ameliorating disease (15). Yoshimura's group found that STAT3, a downstream signaling component for IL- 6 and other cytokines that act through the common $\gamma$-chain receptor, is strongly phosphorylated in the synovial tissue of RA patients compared with tissues from patients suffering from a noninflammatory 
degenerative form of arthritis. The authors found that SOCS-3 transcripts are abundantly expressed in the synovial samples from RA patients, and they noted that in murine models of inflammatory synovitis, STAT3 phosphorylation preceded SOCS-3 expression, consistent with the idea that SOCS-3 is part of a JAK/STAT negative feedback loop. RA-derived synoviocytes transfected with a dominant negative form of STAT3 neither proliferated nor secreted IL-6 in response to serum, suggesting that STAT3 is required for the activation of synovial fibroblasts. In concert with these results, Yoshimura's group observed that forced expression of SOCS-3 inhibited both synovial fibroblast proliferation and IL-6 production (15).

Buoyed by these observations, the investigators attempted to express ectopically either SOCS-3 or a dominant negative form of STAT3 (DNSTAT3) in two animal models of arthritis to suppress the induction of arthritis (15). They injected an adenovirus construct containing either SOCS-3 or DNSTAT3 into the joints of mice susceptible to antigen-induced arthritis and found that joint destruction was prevented in animals expressing either transgene. In the collageninduced arthritis model however, the SOCS-3 construct was at all time points more effective than the DNSTAT3 virus in preventing joint damage. In animals with established inflammatory synovitis, gene transfer of SOCS-3 was still helpful in preventing progression of joint damage, whereas the DNSTAT3 virus conferred little benefit.

This study (15) reinforces the idea that cytokines operating through the $\gamma c$-cytokine receptor are likely important in activating RA synovial fibroblasts. Modulation of the JAK/STAT pathway therefore represents a reasonable strategy for new anti-inflammatory drug development. Specific JAK kinase inhibitors may have a therapeutic role in treating this and other disorders of the immune system especially if their toxicity does not preclude their use. This study also raises a number of questions. Why was the high expression of SOCS-3 transcripts in RA synoviocytes inadequate to quench the persistent activation of STAT3 by the JAK kinases, while forced expression of SOCS-3 by an adenovirus expression vector potently shut down the phosphorylation of STAT3? SOCS proteins are ephemeral and their expression is regulated at multiple levels, including translation and protein degradation $(16,17)$. It will be of interest to determine whether SOCS- 3 protein expression is in any way defective in RA synoviocytes. Another surprise concerns the dominant role of SOCS-3, rather than SOCS-1, in suppressing RA synoviocytes. SOCS-1 was originally cloned for its ability to potently inhibit the IL- 6 pathway in monocytes (2). It will therefore be of interest to examine the expression of SOCS-1 in RA synoviocytes and to determine its effectiveness in terminating inflammation in animal models of arthritis. Another strong prediction of this study is that mice deficient in SOCS proteins would be prone to developing arthritis. Unfortunately, this hypothesis cannot be tested in the case of the SOCS-3 knockout mice as they are embryonic-lethal, but mice lacking both SOCS- 1 and IFN- $\gamma$ are viable for at least 1 year and are said to develop acute synovitis of the small joints (see discussion in ref. 18). Ernst et al. (18) demonstrated a correlation between SOCS-3 expression and phosphorylated STAT3 in activated synoviocytes derived from arthritic joints. This report did not, however, show a causal relationship between abnormal SOCS-3 expression and a propensity to develop arthritis. The capacity of forced expression of SOCS-3 to suppress synovial inflammation should probably be viewed as a general characteristic of SOCS- 3 to inhibit JAK kinase activation, rather than a specific role it might be playing in the pathogenesis of the disease.

The SOCS family may nonetheless represent an interesting class of disease susceptibility genes for disorders arising from abnormal cytokine activity. Hypermethylation and silencing of the SOCS1 locus has recently been observed in hepatic carcinoma cell lines and in primary hepatocellular carcinomas, where dysregulated IL-6 may participate in early stages of this disease (19). Similarly, formation of an IL-6 autocrine loop has been implicated in the pathogenesis of a subset of patients with multiple myeloma (20).
Loss of SOCS-1 may be an important step in sensitizing these tumors to continued IL- 6 stimulation. SOCS-1 may be a critical target for viruses that use multiple strategies to subvert IFN- $\gamma$ production. Virally induced high expression of SOCS- 1 would be expected to shut down IFN- $\gamma$ signaling, sensitizing the infected cell to still more efficient viral replication. Lastly, patients who are treated with IFNs for hepatitis $\mathrm{C}$ viral infections, chronic myelogenous leukemia, and hairy cell leukemia often demonstrate wide variation in their clinical response. Host differences in SOCS expression or differences in tumor expression of SOCS-1 could account for heterogeneity in the clinical response to IFN. SOCS proteins have also been implicated in regulating endocrine hormones such as growth hormone $(\mathrm{GH})$, leptin, and prolactin (21-23). Mice deficient in SOCS-2 develop a form of gigantism that is related to unopposed GH signaling (24). Therefore, we may soon find mutations affecting SOCS protein expression involved in the pathogenesis of a variety of endocrinopathies.

The realization that cytokine circuits cue the termination of their own signal through the induction of SOCS proteins points to this family of proteins as candidate players involved in the initiation and/or propagation of chronic inflammatory diseases. Perhaps the SOCS proteins are the very brakes that we should pull to control diseases of disordered cytokine stimulation.

1. Endo, T.A., et al. 1997. A new protein containing an SH2 domain that inhibits JAK kinases. Nature. 387:921-924.

2. Starr, R, et al. 1997. A family of cytokine-inducible inhibitors of signalling. Nature. 387:917-921.

3. Rottapel, R., Ilangumaran, S., and De Sepulveda, P. 2000. On the road to destruction: suppression of protein tyrosine kinase signalling by SOCS family proteins. In Protein kinase functions. J Woodgett, editor. Oxford University Press. 246-276.

4. Yasukawa, H., et al. 1999. The JAK-binding protein JAB inhibits Janus tyrosine kinase activity through binding in the activation loop. $E M B O J$. 18:1309-1320

5. Alexander, W.S., et al. 1999. SOCS1 is a critical inhibitor of interferon gamma signaling and prevents the potentially fatal neonatal actions of this cytokine. Cell. 98:597-608.

6. Marine, J.C., et al. 1999. SOCS1 deficiency causes a lymphocyte-dependent perinatal lethality. Cell. 98:609-616.

7. Metcalf, D., Di Rago, L., Mifsud, S., Hartley, L. and Alexander, W.S. 2000. The development of fatal myocarditis and polymyositis in mice heterozygous for IFN-gamma and lacking the SOCS 1 gene. Proc. Natl. Acad. Sci. USA. 97:9174-9179. 
8. Marine, J.C., et al. 1999. SOCS3 is essential in the regulation of fetal liver erythropoiesis. Cell. 98:617-627.

9. Auernhammer, C.J., and Melmed, S. 2001. The central role of SOCS-3 in integrating the neuroimmunoendocrine interface. J. Clin. Invest. 108: $1735-1740$.

10. Keffer, J., et al. 1991. Transgenic mice expressing human tumour necrosis factor: a predictive genetic model of arthritis. EMBO J. 10:4025-4031.

11. Elliott, M.J., et al. 1993. Treatment of rheumatoid arthritis with chimeric monoclonal antibodies to tumor necrosis factor alpha. Arthritis Rheum. 36:1681-1690.

12. Moreland, L.W., et al. 1997. Treatment of rheumatoid arthritis with a recombinant human tumor necrosis factor receptor (p75)-Fc fusion protein. N. Engl. J. Med. 337:141-147.

13. Alonzi, T., et al. 1998. Interleukin 6 is required for the development of collagen-induced arthritis. $J$. Exp. Med. 187:461-468.
14. Ohshima, S., et al. 1998. Interleukin 6 plays a key role in the development of antigen-induced arthritis. Proc. Natl. Acad. Sci. USA. 95:8222-8226.

15. Shouda, T., et al. 2001. Induction of the cytokine signal regulator SOCS3/CIS3 as a therapeutic strategy for treating inflammatory arthritis. $J$. Clin. Invest. 108:1781-1788.

16. Gregorieff, A., Pyronnet, S., Sonenberg, N., and Veillette, A. 2000. Regulation of SOCS-1 expression by translational repression. J. Biol. Chem. 275:21596-21604.

17. Kamura, T., et al. 1998. The Elongin BC complex interacts with the conserved SOCS-box motif present in members of the SOCS, ras, WD-40 repeat, and ankyrin repeat families. Genes Dev. 12:3872-3881.

18. Ernst, M., et al. 2001. Defective gp130-mediated signal transducer and activator of transcription (STAT) signaling results in degenerative joint disease, gastrointestinal ulceration, and failure of uterine implantation. J. Exp. Med. 194:189-203.

19. Yoshikawa H, et al. 2001. SOCS-1, a negative reg- ulator of the JAK/STAT pathway, is silenced by methylation in human hepatocellular carcinoma and shows growth-suppression activity. Nat. Genet. 28:29-35.

20. Kawano, M., et al. 1988. Autocrine generation and requirement of BSF-2/IL- 6 for human multiple myelomas. Nature. 332:83-85.

21. Bjorbaek, C., et al. 1998. Identification of SOCS3 as a potential mediator of central leptin resistance. Mol. Cell. 1:619-625.

22. Pezet, A., Favre, H., Kelly, P.A., and Edery, M. 1999. Inhibition and restoration of prolactin signal transduction by suppressors of cytokine signaling. J. Biol. Chem. 274:24497-24502.

23. Tollet-Egnell, P., Flores-Morales, A., StavreusEvers, A., Sahlin, L., and Norstedt, G. 1999. Growth hormone regulation of SOCS-2, SOCS3 , and CIS messenger ribonucleic acid expression in the rat. Endocrinology. 140:3693-3704.

24. Metcalf, D., et al. 2000. Gigantism in mice lacking suppressor of cytokine signalling-2. Nature. 405:1069-1073. 